\title{
Beam-beam interaction studies at the Cornell Electron Storage Ring
}

\author{
M. G. Billing* and J. A. Crittenden ${ }^{\dagger}$ \\ Laboratory for Elementary-Particle Physics, Cornell University, Ithaca, New York 14853-8001, USA
}

(Received 1 September 2006; published 20 December 2006)

\begin{abstract}
The Cornell Electron Storage Ring (CESR) operates with $2 \mathrm{GeV}$ multibunch electron and positron beams in a single beam pipe. Electrostatic separators are used to separate the two counterrotating beams at the parasitic crossings. When the beam energy was lowered from $5 \mathrm{GeV}$ in 2003 , the strength of the beambeam interaction became a more important factor in beam-current limitations, resulting in extensive experimental and modeling studies of their characteristics. The CESR lattice design procedure has been modified recently to account explicitly for their dynamic consequences. We describe our modeling of the beam-beam interaction, experimental validation techniques, and investigations into compensation strategies.
\end{abstract}

DOI: 10.1103/PhysRevSTAB.9.121001

PACS numbers: 29.20.Dh, 41.75.Ht

\section{INTRODUCTION}

Optical distortions due to the beam-beam interaction are an important factor in the lattice design of many storage rings presently in operation [1]. Beam-beam effects are a major consideration in the design of linear colliders as well $[2,3]$. In this article, we describe measurements of the optical distortions arising from the beam-beam interaction at the Cornell Electron Storage Ring (CESR) and compare them to modeling studies. CESR operates countercirculating beams in a single beam pipe, employing electrostatic separators to separate them at the parasitic crossing points. The linear optics are constrained to be similar for the electron and positron beams. Following two decades of operation at $5 \mathrm{GeV}$ beam energy for studies of bottom quark bound states, CESR converted to $2 \mathrm{GeV}$ operation in 2001 for the purpose of investigating bound states of charm quarks, naming the project CESR-c [4]. This reduction of beam energy resulted in increased relative contributions of the beam-beam force. The beam-beam interaction has been found to have considerable consequences for the optics [5] and for injection aperture [6]. We report here on measurements of the closed-orbit distortions induced by the parasitic crossings, on operational strategies used to mitigate focusing errors, including those arising from the beam-beam interaction at the collision point, and on the implementation and tests of a local betatron phase compensation algorithm [7].

CESR-c operated in early 2006 at a beam energy of $2.085 \mathrm{GeV}$ with three bunches $4.2 \mathrm{~m}$ apart in each of eight trains separated by 75.6 or $79.8 \mathrm{~m}$. The ninth possible equally spaced train is omitted for purposes of ion clearing. During luminosity operation, the total beam current in both beams was limited to about $2.5 \mathrm{~mA} /$ bunch, i.e., $120 \mathrm{~mA}$. Appreciably higher currents can be injected when the beams are not in collision. Single-bunch currents as high

\footnotetext{
*Electronic address: mgb@lepp.cornell.edu

${ }^{\dagger}$ Electronic address: critten@lepp.cornell.edu
}

as $8 \mathrm{~mA}$ have been reached for a single electron bunch injected into a full load of positrons. Good luminosity lifetime has also been obtained with single-bunch collisions at bunch currents about twice as high as during multibunch operation. The importance of the bunchspacing pattern for beam lifetimes, luminosity lifetimes, and injection limits have motivated extensive investigation into the distortions of the lattice functions caused by the parasitic crossings. We designate the location of a bunch in the fill pattern by its train number (1-9) and its bunch number within the train (1-5), where like-numbered electron and positron bunches collide at the interaction point.

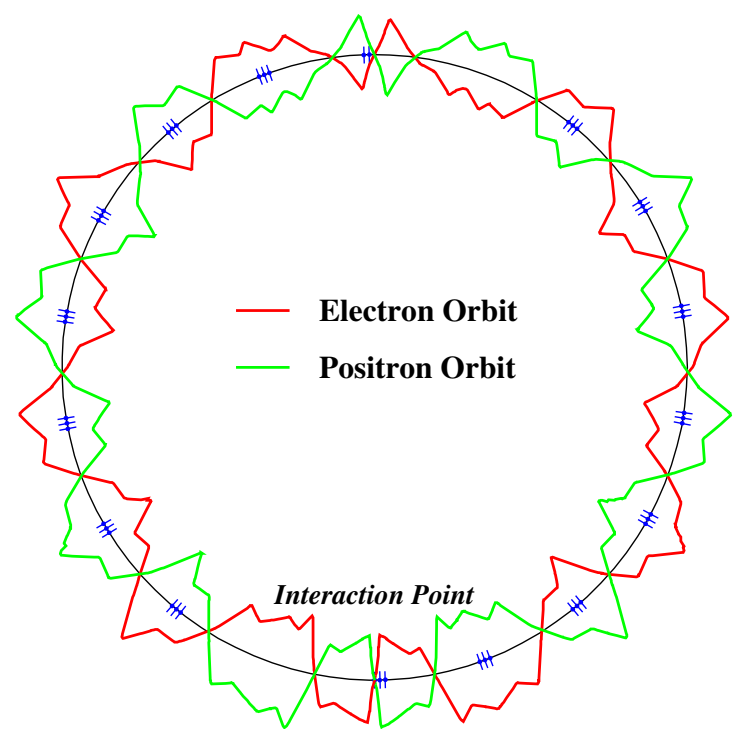

FIG. 1. (Color) Electrostatic separators establish closed-orbit waves separating the electron and positron beams at the positions of the parasitic crossings (blue lines). The parasitic crossing pattern shown here corresponds to the particular case of the first bunch of the first electron train against the counterrotating positron configuration of three bunches (1-3) in each of eight trains (1-8). This was the operational configuration for CESR-c in early 2006. 
As Fig. 1 illustrates for three bunches in each of eight trains, each electron and positron bunch suffers a beambeam interaction at 47 points around the ring, as well as at the primary interaction point. In this figure, the positrons circulate in the clockwise direction. The beam separation at each crossing point is determined by the pretzel orbit induced by vertical and horizontal electrostatic separators, and ranges between 20 and $35 \mathrm{~mm}$. The separation is vertical at the crossing point diametrically opposed to the collision point and horizontal at the remaining parasitic crossing points.

\section{BASICS OF THE BEAM-BEAM FORCE}

It is useful first to review the lowest order multipole effects coming from the beam-beam interaction at both the interaction point and the parasitic crossings. Although some of the following effects are particular to CESR-c and its pretzel-separation orbits, the majority of them are common to other colliders having parasitic crossings. Since the effects of the beam-beam interaction from the interaction point and from the parasitic crossings are quite different, we review them separately.

At the interaction point, particles oscillating with small amplitudes see a linear focusing force in both transverse planes. Particles with larger amplitudes will, on average, be subject to a smaller restoring force from the other beam. As long as one beam is aligned with the center of the other beam, there will be no average deflection in either plane.

At each parasitic crossing, each beam passes the other with a separation much greater than the beam sizes. In most parasitic crossings in CESR-c, this separation is horizontal, but at a few parasitic crossings, such as in the cluster of parasitic crossings diametrically opposite to the interaction point, there is vertical separation in addition. For particles executing small-amplitude oscillations about their central orbit, there is an average deflection toward the other beam, an average defocusing force in the plane of the separation, and an average focusing force in the other plane. For particles oscillating with larger amplitudes in the plane of separation, the in-plane deflection increases on the average and the focusing/defocusing effects for both planes increase on the average. Particles oscillating with large amplitudes out of the plane of separation will be subject to much less change in the average deflection and to a reduced defocusing/focusing force in/out of the plane of separation. Until the amplitude of oscillation causes the particle to approach within a few horizontal sigma of the core of the counterrotating beam, the nonlinearities of the beam-beam force will be much smaller at the parasitic crossings than those generated at the interaction point.

The beam-beam interaction alters the orbit of each beam, owing to the deflection arising at each parasitic crossing. Since the average deflection scales to lowest order linearly with the current in the countercirculating beam, the orbits also deform proportionally in lowest order.
When one particle passes by or through the other beam, the deflection is attractive, making the beam-beam force antisymmetric about the center of the opposite bunch. As a result, since the pretzels in CESR-c themselves are nearly antisymmetric about the east-west axis of the storage ring, the orbit distortions of the two beams will be nearly equal and opposite, implying that there should be relatively little change in the separation at the interaction point as the beam current increases. However, in general there may be some change in the horizontal crossing angle with beam current.

At each parasitic crossing and at the interaction point, particles experience a gradient to the beam-beam force. Unlike a quadrupole magnet, the gradients of the force in the horizontal and vertical planes are not equal and opposite. Since the deflection arising from the beam-beam interaction is antisymmetric, the gradient of this force is symmetric about the center of the opposite beam. This means that, if both beams have the same charge and cross section, the focusing forces will be equal for the two beams in both the horizontal and vertical planes. These average gradients cause tune shifts and beta waves proportional to the current in the opposing beam. Since CESR-c operates close to the half-integer resonance for the horizontal tune, the focusing errors are particularly important for the horizontal plane.

\section{CONSEQUENCES OF THE BEAM-BEAM INTERACTION FOR CESR-c OPTICS}

A detailed software package has been developed to model the CESR-c optics [8]. The optical effects of the parasitic crossings in the CESR storage ring have been computed in a weak-strong approximation, where the beam functions of one of the beams (the positron beam) are held fixed, and the optical distortions due to the beam-beam interaction for the closed orbit of a single electron are calculated. The model for the beam-beam interaction uses the Bassetti-Erskine complex error function formula $[9,10]$, in which a Gaussian transverse shape in the strong beam is assumed. The calculated distortion differs for electrons in different bunches of the electron beam, owing to the differing pattern of parasitic crossings.

Although CESR-c typically operates with an electron beam current nearly as high as the positron beam current, the weak-strong approximation is justified for the parasitic crossings, since perturbations of the beam size and position in the strong beam are small relative to the beam-beam separation, so the long-range force is scarcely modified. Our modeling of the beam-beam force at the interaction point uses a beam size corresponding to the bunch current under consideration. Operationally, we have observed no evidence for any coherent beam-beam motion up to our present operating current levels.

Orbit distortions are one measurable consequence of the beam-beam interactions which can be used to assess the 
accuracy of the model. The upper plot in Fig. 2 shows the horizontal electron-positron orbit differences at the crossing positions for an electron populating the first bunch of the first train in the electron beam, where an operating current level of $2.5 \mathrm{~mA} /$ bunch in positron bunches $1-3$ of trains 1-8 has been assumed. The distance from the interaction point is given in the positron flight direction. The lower plot shows the calculated horizontal angular deflections induced at the parasitic crossings. The deflections in this case range between 1 and $4 \mu \mathrm{rad}$. The two points showing anomalously low transverse kicks correspond to the two parasitic crossings near the crossing point diametrically opposed to the interaction point, where there is additional vertical separation.

Our model of the CESR-c optics has shown that the consequences of the beam-beam interaction for particles in the core of the beam are severe. Figure 3 shows the modeled maximum value of the horizontal beta function for electrons in train 1 at any point in the ring as a function of positron bunch current for the operational CESR-c lattice of early 2006, when a configuration of three bunches in each of eight trains was used. At a bunch current of $4 \mathrm{~mA}$, this maximum value of the horizontal beta function increases to $110 \mathrm{~m}$ from its design value of $45 \mathrm{~m}$. Operationally we find an empirical beam-beam interaction current limit of $2.5 \mathrm{~mA}$, corresponding to an increase in the
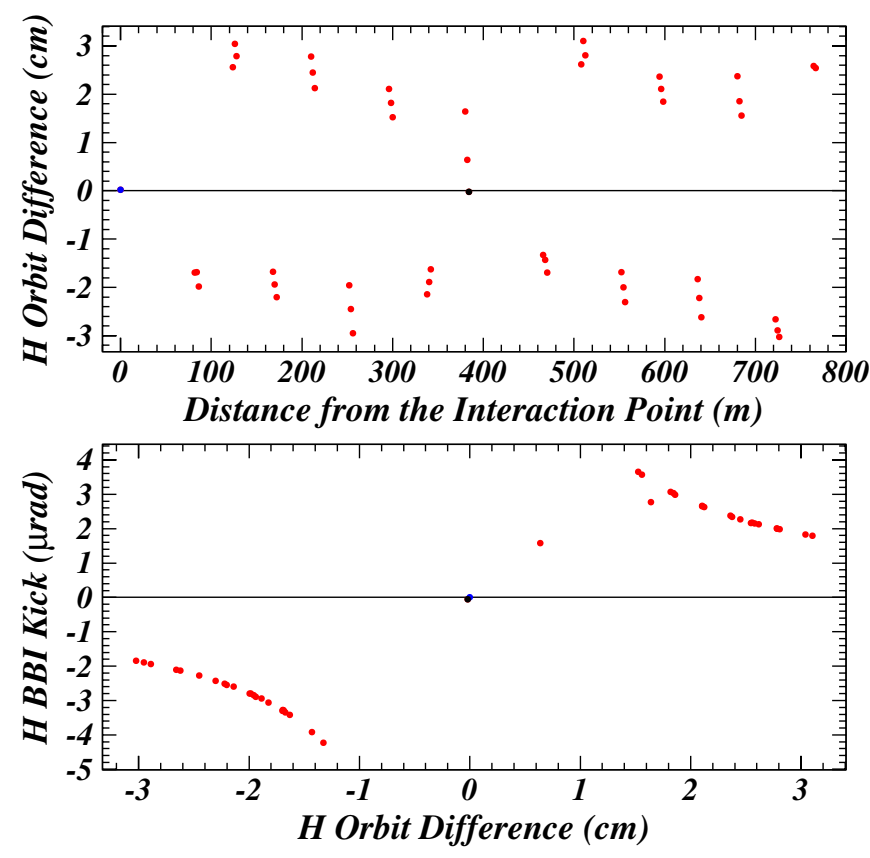

FIG. 2. (Color) Modeled horizontal positron-electron orbit differences at the crossing points in the CESR-c model of the operational $8 \times 3$ configuration as a function of their position around the ring (upper plot) and the horizontal kicks resulting in the Bassetti-Erskine approximation as a function of this orbit difference (lower plot). The black point indicates the crossing diametrically opposed from the main interaction point (shown in blue), where the separation is vertical.

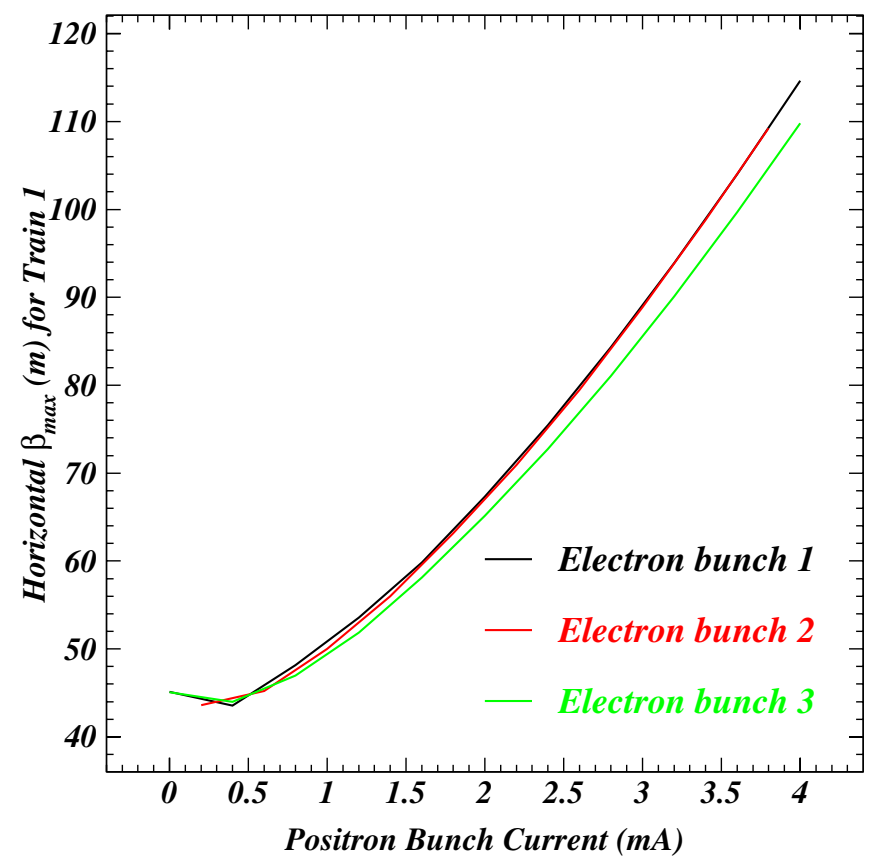

FIG. 3. (Color) Modeled maximum value of the electron horizontal beta function for the central orbit in the CESR-c lattice as a function of the positron bunch current.

maximum beta value of about $70 \%$. The optical distortion generally differs for each electron bunch owing to the different parasitic crossing pattern it encounters, but the dominant effect results from the beam-beam interaction at the interaction point and is common to all bunches.

In December 2005, the lattice design optimization algorithm was modified to take the parasitic crossings into account using the weak-strong approximation. This approach was chosen since parasitic crossings with large separations relative to the beam sizes produce similar focusing errors for particles at both large and small amplitude. The optimized lattice was designed for a specific bunch (train 1 bunch 3) and a specific operating current ( $2.5 \mathrm{~mA} /$ bunch). A design procedure was developed which handled the electron and positron optics symmetrically, each in the weak-strong approximation. Figure 4 shows calculations of the dynamic aperture at the interaction point based on tracking electrons for 500 turns through the modeled optics. The aperture is calculated at the interaction point in units of rms beam size. The vertical beam size is the value assuming full emittance coupling in the two planes. A slight increase in electron horizontal aperture is observed for the case with $2.5 \mathrm{~mA} /$ bunch positron current. This calculation demonstrates that the design procedure indeed succeeded in improving the aperture at the design current while maintaining acceptable aperture for lower currents. Optics obtained with this new lattice design algorithm typically exhibit a substantial increase in the horizontal aperture for low vertical amplitudes, owing to the proximity of the horizontal tune to the half-integer resonance. 

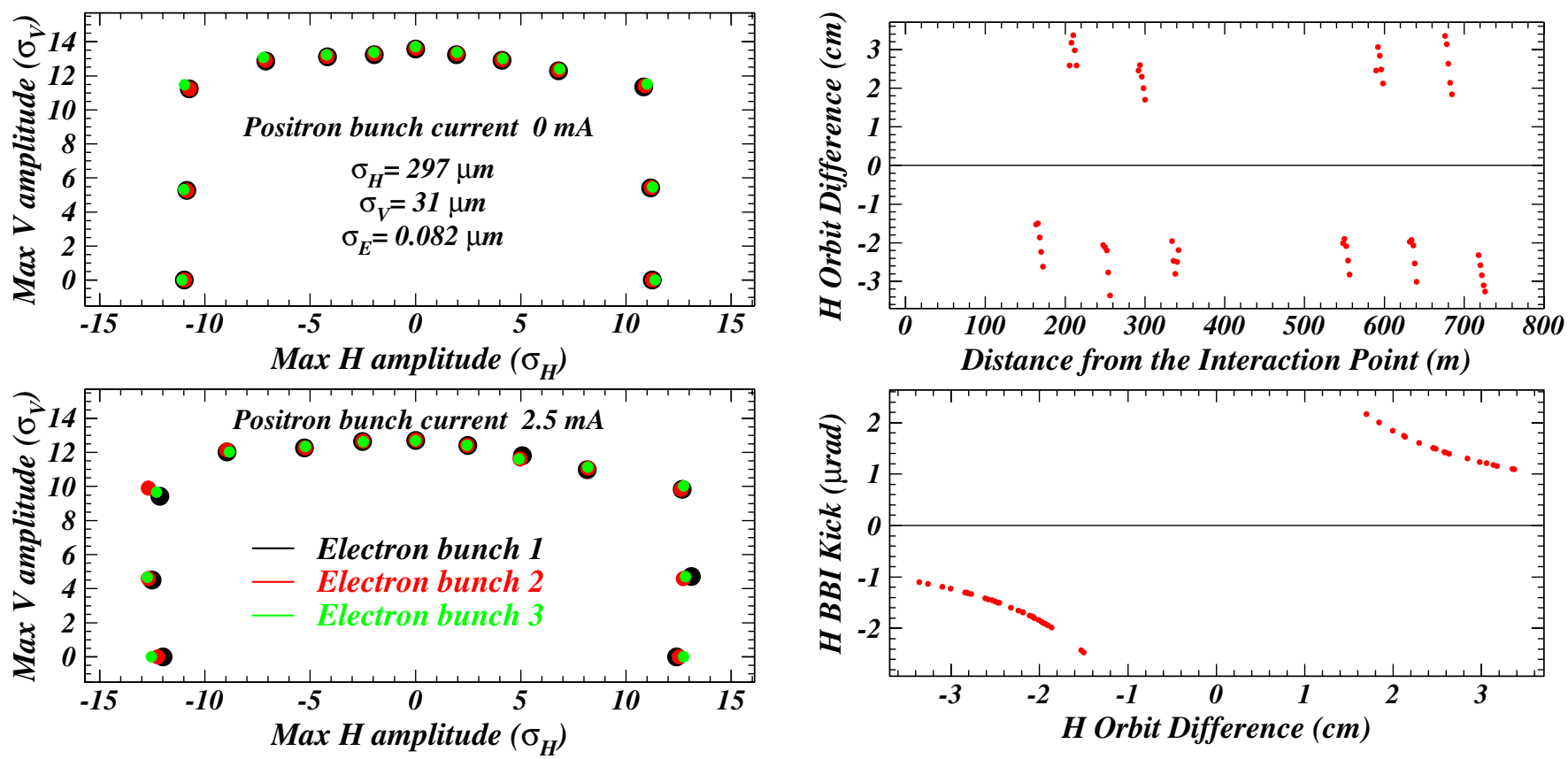

FIG. 4. (Color) Comparison of the electron dynamic aperture in the distortion-free optics (upper plot) to that in the optics distorted by $2.5 \mathrm{~mA} /$ bunch in the positron beam (lower plot). The lattice design procedure developed to account for the effects of the parasitic crossings yields an improved aperture at the operating current.

\section{MEASUREMENTS OF ORBIT DISTORTIONS}

We performed measurements of orbit distortions induced by the parasitic crossings in the absence of any collisions at the interaction point to obtain quantitative tests of our modeling of the beam-beam kicks in a weakstrong approximation. A positron beam consisting of bunches $1-5$ in trains 2-6 was used to avoid creating parasitic crossings in the region of the beam-position monitors which had been upgraded to allow exclusive, gated, measurements of the electron orbit. Figure 5 shows the modeled orbit separations and kicks for the first bunch of the first electron train, as was used in the measurement. The positron bunch current was $1.6 \mathrm{~mA}$.

A comparison of the modeled orbit distortion to the 11 beam-position monitor measurements in this region is given in Fig. 6. The red line shows the horizontal electron orbit in the presence of the positron beam with the undistorted orbit subtracted. Orbit deviations up to $0.4 \mathrm{~mm}$ are observed and reproduced in the model to an accuracy of about $0.05 \mathrm{~mm}$.

We performed several systematic checks of this measurement: (i) the current in the single electron test bunch was reduced from 2 to $1 \mathrm{~mA}$ with negligible effect, validating the weak-strong approximation; (ii) the positron bunch current was raised to $1.9 \mathrm{~mA}$ and lowered to $0.9 \mathrm{~mA}$, testing the scaling properties of the model; (iii) the orbit separation was reduced by $15 \%$, increasing the

FIG. 5. (Color) Modeled orbit separations at the parasitic crossings and resulting beam-beam interaction kicks in the $5 \times 5$ configuration employed to measure the orbit distortions shown in Fig. 6.

orbit distortion in the same manner for both measurement and model. The modeled orbit distortion was found to be qualitatively similar for a wide variety of positron bunch configurations, as the effects of the parasitic crossings tend to add coherently, but was found to be quite different when

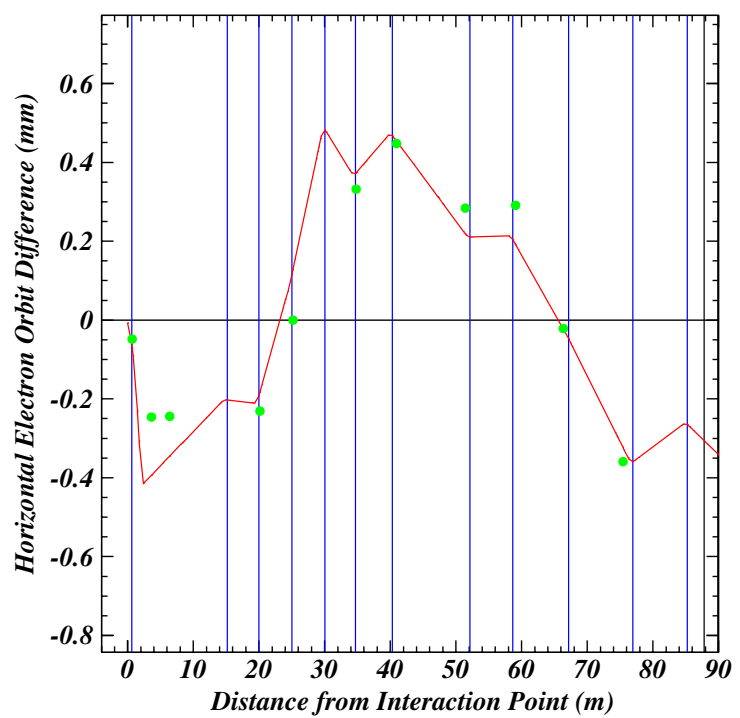

FIG. 6. (Color) Comparison of the measured difference between the horizontal electron orbits at the 11 beam position monitor positions (green dots) with and without the positron beam to the modeling result (red line). The blue lines show the positions of the quadrupole magnets and the nearby superconducting wiggler magnet is shown as well (black line). 
only train 9 was filled. This difference was also validated by the orbit measurements. All observed comparisons exhibited an accuracy comparable to that shown in Fig. 6.

\section{STRATEGY FOR LOCAL BEAM-BEAM INTERACTION COMPENSATION}

The only operational tool for compensating the beambeam interaction in CESR-c optics has heretofore been a global tune adjustment employing all quadrupole magnets. During luminosity running, the tunes are adjusted to provide the best beam lifetimes. Since the tune shift in the core of the beam due to the interaction point is typically five times greater than that from all the parasitic crossings combined, the primary operational adjustment is to reduce the horizontal and vertical tunes globally as the colliding current increases.

This global adjustment results in local distortions of the phase and beta functions. To reduce these distortions, we have developed a compensation method based on local phase and beta function corrections using the eight quadrupole magnets surrounding each of the clusters of parasitic crossings associated with crossing of individual trains.

Since CESR-c has multiple trains of multiple bunches with a variety of spacings, the orbit differences and the focusing errors vary from bunch to bunch. At each lobe of the pretzel, the bunches pass by each other at parasitic crossings which are of similar betatron phase advance. The small betatron phase advance between the parasitic crossings at each lobe causes the orbit and beta-function errors to add approximately coherently. This suggests that there will be a significant common set of errors for all of the bunches arising from the parasitic crossings. Since the beam-beam interaction at the main interaction point is much stronger than that from any single parasitic crossing (and can be larger than that from the full set of parasitic crossings), the main interaction point itself produces a common error for all bunches. It is possible to take advantage of the flexibility of lattice design provided by the many quadrupole magnets in CESR-c to correct the average set of errors felt by the ensemble of bunches and this idea is the premise for the correction scheme described below. After the best overall correction is applied, there will remain residual differences between bunches, which, in the best case, will be distributed, leaving a small net error about zero. The validity of this last assertion depends on exactly what type of correction is applied.

There are two basic types of particle motion for which the corrections may be applied. The most obvious case corresponds to a particle traveling near the core of the beam. Such particles are responsible for the characteristics of the luminosity performance of the collider (assuming the beam separation at the interaction point is kept small.) This beam-beam correction is generally relatively easy to calculate by effectively using the local gradients of the beam-beam kicks for the central particle. The second case to consider is for particles oscillating with large amplitudes in the range of 4-7 sigma of the particle distribution; the properties of these particles determine the lifetimes of the beams. For this case, a weak-strong calculation can be performed by tracking large amplitude particles through the ring with the beam-beam interaction included at the interaction point and all parasitic crossings. From the tracking, average envelope and phase functions can be inferred and their deviation from the design can be corrected. Particles oscillating with larger amplitudes in the plane of the pretzel separation require a greater correction at the parasitic crossings, since they sample a larger average gradient. Out of the plane of separation, the correction should be similar to that for the small-amplitude particles. The corrections required by large-amplitude particles at the interaction point are much smaller in both planes than those required by small-amplitude particles.

Our strategy has been to develop an algorithm which will provide some compensation for the most important optical errors. The choice has been made to compensate the average phase advance, and the sinelike and cosinelike beta-wave errors produced in both the horizontal and vertical planes for the set of parasitic crossings in each pretzel lobe. There are thus a total of six parameters to be corrected for each pretzel lobe. This scheme is possible since there are eight independently controlled quadrupoles in the region containing the parasitic crossings for a single lobe of the pretzel. The use of locally closed corrections at one set of parasitic crossings reduces the effect of optical errors in distant parts of the ring. The result is that the beta errors at those parasitic crossings are linear over a larger range of charge per bunch. It also reduces the sensitivity of the corrections to changes in the betatron tunes. Inasmuch as the bunch currents and sizes of the two beams are similar, as is the case in CESR-c, this correction will be equally effective for each beam.

The algorithm chosen does not correct the orbit errors caused by the beam-beam interaction. This choice was motivated by the fact that the beam-beam interaction deflections alone will tend to produce an angle error at the interaction point and can be easily corrected with the pretzel controls. In addition, this algorithm's local compensation of the changes in the phase advance and betafunction reduces the net focusing errors in the region between the horizontal separators, which spans a large number of parasitic crossings. This minimizes a class of distortions which can produce horizontal separation at the interaction point.

\section{CONTROLS FOR THE IMPLEMENTATION OF THE COMPENSATION ALGORITHM}

For each of the pretzel lobes, six software control knobs were created using the eight quadrupoles associated with that particular lobe. For a given lattice, the scaling coefficients for the eight quadrupoles were determined for each 
of the six control parameters. These coefficients are calculated to produce a linear correction of the prescribed parameter in some absolute units, for example, degrees of phase advance change for some particular change in the control knob, while holding the other parameters constant. Since there are more quadrupoles than control functions, the coefficients can be chosen to minimize the changes to the quadrupole strengths. Since this correction is as close to the source of the errors as possible, it is much less sensitive to overall changes in tunes or errors generated elsewhere. For the 18 sets of parasitic crossings (including the set that contains the interaction point) there are a total of 108 separate control knobs required. These are defined in an accelerator control system node named CSR PCBETING. Designing these knobs for a set of optics is a relatively slow and computer intensive process, but only one set of controls needs to be generated for a particular set of optics. The set of CSR PCBETING knobs is then used as the basis set of control parameters for the optics. Given a pretzel amplitude, a number of trains and a number of bunches per train, the required corrections to be applied to the CSR PCBETING knobs are calculated per unit current in the counterrotating beam for each pretzel node. Since this latter calculation is relatively fast, this correction vector may be changed on short notice as different pretzel amplitudes or bunch patterns are tried.

The compensation software calculates the required corrections for the effects of the beam-beam force at the parasitic crossings on the central trajectory of the weak beam and therefore computes the beam-beam compensation only for small-amplitude particles. Since the ultimate interest is to improve the lifetimes of the beams, it is necessary to understand the average errors experienced by the large-amplitude particles as well. The average errors from the parasitic crossings will be similar for smallamplitude and large-amplitude particles. However, at the interaction point, the average errors experienced by the large-amplitude particles will be very different from those experienced by small-amplitude particles, because largeamplitude motion samples regions of lower gradient. Since the corrections for large-amplitude particles at the main interaction point were not calculated, we chose instead to determine them experimentally. This was accomplished by colliding single bunches and turning on closed, pulsed bumps which displaced the beams to large amplitude only near the injection point of the ring for four revolutions with no displacement at the interaction point. All six corrections for the pretzel crossing at the interaction point were adjusted to obtain the best lifetime with the pulsedbump firing. In principle, since the pulsed bumps are exploring the horizontal aperture limits in a particular section of the ring, an optimally improved pulsed bump lifetime represents the least distortion of the optics for large-amplitude particles in that section of CESR-c. Since generally the particles that have the poorest lifetimes are lost in the neighborhood of beam-beam resonances, reducing the phase advance and beta distortions in one section of the ring implies a reduction in the overall beam-beam interaction resonance driving terms.

The present version of the software can use either the design pretzel amplitude or the command values of the horizontal separator voltages to determine the orbits of the electron and positron beams. It also requires as input the specific bunch and train pattern that is to be corrected. These two sets of parameters are each necessary, since they change the separation of the counterrotating beam and the locations of the parasitic crossings. The program steps through each lobe of the pretzel. In each lobe, it calculates the changes in phase advance and beta functions as seen by one of the bunches in the train (usually one of the center ones) arising from a differential change in the charge in the counterrotating bunches. It then computes the corresponding command changes for all of the CSR PCBETING control knobs in that cluster of parasitic crossings to correct these optical errors. After cycling through the 18 possible clusters of parasitic crossings, it creates a command file for loading the control coefficients. This file allows the definition of a set of global knobs (in the CSR PCCMDING accelerator control system node) to adjust all of the 108 control knobs in parallel. The computing time necessary for the calculation and loading a new set of coefficients when changing the bunch pattern and horizontal separator settings is about ten minutes.

During operations, a control program reads the total current in both beams and applies a scaled correction to all of the 108 CSR PCBETING knobs. This choice is motivated by the fact that the beam-beam interaction focusing effects scale proportionally to the beam current for all parasitic crossings and for at least the horizontal focusing at the interaction point. The vertical focusing at the interaction point generally increases in proportion to the beam current until the vertical tune shift parameter saturates at high current. Therefore, the control commands are chosen to scale with the total stored beam current.

The beam-beam compensation control software has two basic modes of operation. The first is a manual control mode where the operator can adjust the beam current level which is to be compensated. In the second mode of operation, the software automatically adjusts the commands to the PCBETING node proportionally to the average current in the two beams. Regardless of which of these two modes of operation is in use, there are controls within the CSR PCCMDING node that scale the overall corrections for the horizontal and vertical tunes and beta-waves. There are also knobs which allow the adjustment of the main interaction point compensation separately. The use of the control software requires a multistep procedure to establish operating conditions. Specific procedures have been defined for three modes of colliding-beam operations: (i) topping off positrons and filling electrons from zero cur- 
rent, (ii) topping off both beams, and (iii) single-set operations, which are to be used for quasicontinuous injection, whereby all ring element settings remain unchanged when switching from electron to positron injection and vice versa. The first of these operating modes is more problematic for developing a stable set of operating conditions, because of the larger variation of quadrupole strengths during the routine filling/colliding cycle. As an example, we describe here the procedure to establish the operating conditions for the third of the operating modes, the singleset mode.

Step 1: Fill to colliding conditions at approximately $3 / 4$ of the peak operating current.

Step 2: Adjust the CSR PCCMDING element, which controls the strength of the current-dependent correction, from an initial setting of zero toward the present average bunch current. During this step, it is necessary to adjust the tunes of the beams to maintain good lifetimes. Since this reverses the manual tune adjustment required for Step 1 to maintain the lifetimes of the colliding beams during filling, this procedure transforms the optics back to the original optics plus the beam-beam interaction corrections necessary for the operating current.

Step 3: Switch the program to current tracking mode, where it will change all of the CSR PCBETING elements in proportion to the average beam current. At this point, the dynamic partial compensation of the beam-beam interaction will be in effect.

\section{MODELING THE EFFECTIVENESS OF THE BEAM-BEAM INTERACTION COMPENSATION}

The calculation of the beam-beam interaction compensation coefficients at the parasitic crossings was carried out by optimizing the quadrupole currents so as to minimize the phase distortions in the core of the beam. Since the corrections are to be used in collision and in top-off mode, compensation for the distortions arising at the interaction point was provided as well, employing the set of quadrupoles surrounding the interaction point. These coefficients were determined empirically, using the procedure described in Sec. VI, which optimized beam lifetimes in a single-bunch collision configuration. The correction coefficients for the beam-beam interaction at the interaction point and at the near-interaction-point parasitic crossings were linearly superposed to get the full correction. Using this set of correction coefficients, the CESR-c model then served to assess the expected effectiveness of the algorithm, both for the beam functions for the central orbit and for the large-amplitude tails of the beam as determined by tracking calculations of dynamic aperture. Figure 7 shows the improvement in the maximum value of the beta function for particles in the core of the beam over that shown in Fig. 3, where solely the global tune correction was employed. The level of beta distortion reached at $2.5 \mathrm{~mA} /$ bunch with the global tune correction is compa-

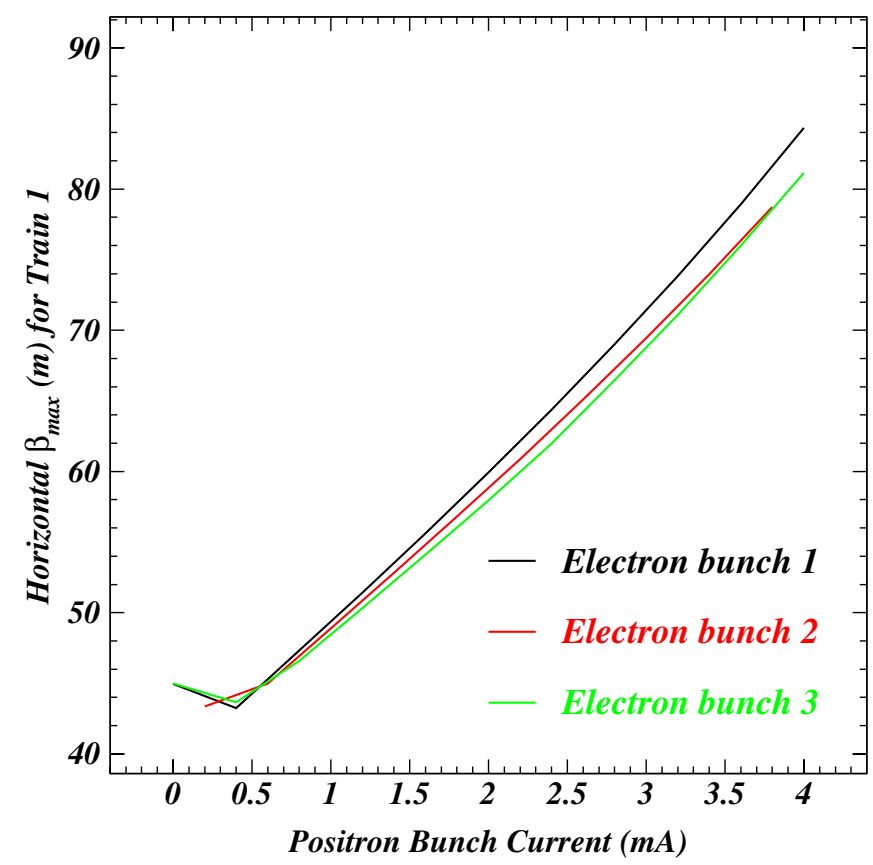

FIG. 7. (Color) Modeled maximum value of the electron horizontal beta function for the central orbit in the CESR-c optics as a function of the positron bunch current when ameliorated by the local beam-beam interaction compensation algorithm. Comparison with Fig. 3 yields the advantage of the local beam-beam interaction correction over a global tune correction alone.
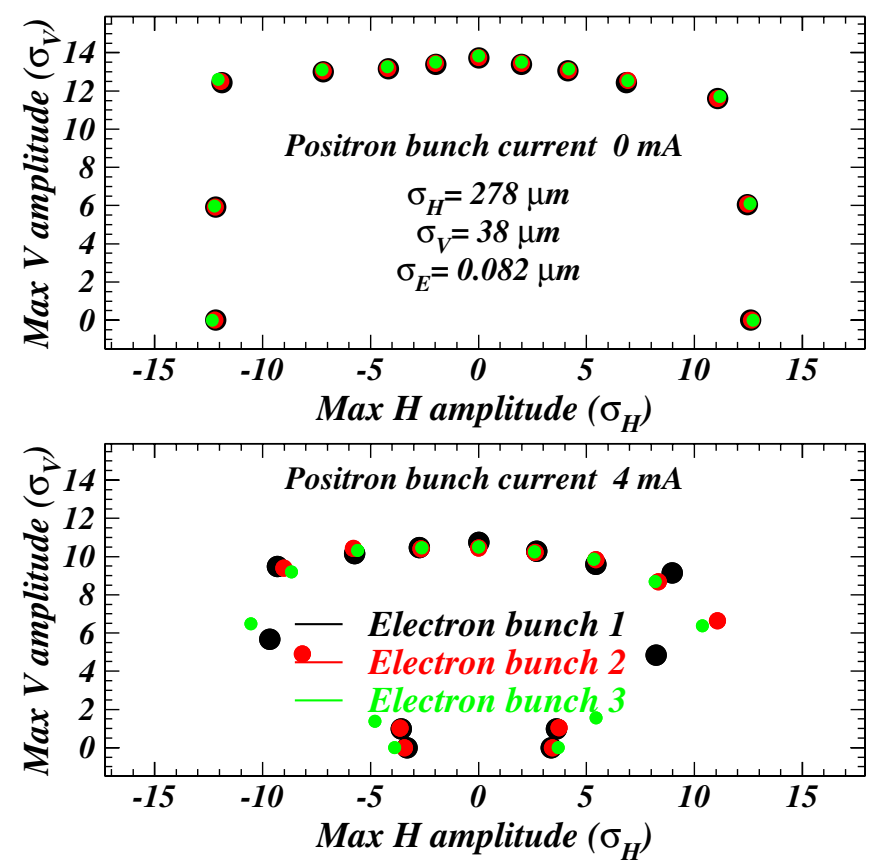

FIG. 8. (Color) Comparison of the modeled electron dynamic aperture in the distortion-free optics (upper plot) to that in the optics distorted by $4.0 \mathrm{~mA} /$ bunch in the positron beam (lower plot), where solely a global tune correction is used to partially compensate the beam-beam interaction. 


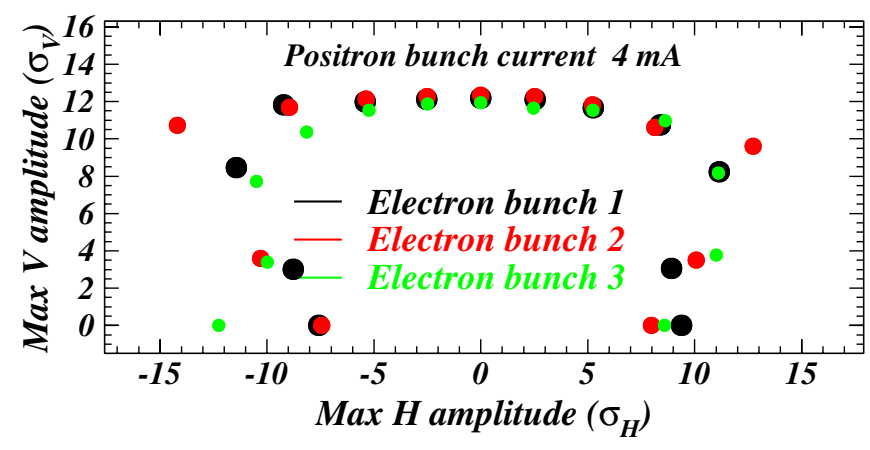

FIG. 9. (Color) The calculated electron dynamic aperture in the optics distorted by $4.0 \mathrm{~mA} /$ bunch in the positron beam, where the effect of the local beam-beam interaction compensation algorithm has been included in the model. Comparison with Fig. 8 allows assessment of the degree of improvement afforded by the local beam-beam interaction correction over a global tune correction alone.

rable to the level reached at $3.6 \mathrm{~mA} /$ bunch when the local beam-beam interaction compensation is used. The apparent undercorrection of the beta distortion is a consequence of using the beam lifetime (i.e. dynamic aperture) as the criterion for determining the correction coefficients, since the average beam-beam kick at the interaction point is much smaller for large-amplitude particles than that for particles on the central orbit.

We also employed our model to estimate the effects on the large-amplitude tails by tracking particles generated off-axis at the interaction point. Figure 8 shows the dynamic-aperture calculation for the bunches in electron train 1 with a positron bunch current of $4.0 \mathrm{~mA} /$ bunch in bunches $1-3$ of trains $1-8$, where only a global tune correction has been applied. This bunch current value, which is higher than the operational limit, was chosen to reach a 3-sigma horizontal aperture for electron trajectories having small vertical amplitude. When the local compensation including the empirically tuned interactionpoint-compensating coefficients is used, the aperture increases to eight sigma, as shown in Fig. 9. It is notable that while the empirical tuning of the coefficients tested the aperture using only the pulsed bump at the electron injection point, the model extends the result to the entire ring. This confirmation by the model of the observed improvement in beam lifetimes obtained during the empirical tuning of the correction coefficients, together with its ability to reproduce the orbit distortions as described in Sec. IV, served to establish our confidence in the reliability of its compensation calculations.

\section{OPERATIONAL EXPERIENCE}

During March and April of 2006, machine studies of this partial compensation scheme were carried out in CESR-c. The initial portion of these studies checked the effectiveness of a subset of the CSR PCBETING control knobs and verified that they functioned as designed. The studies continued with determination of the compensation settings for the interaction point which produce the best lifetime for colliding single bunches, as described in Sec. VI.

The second phase of the machine studies program was an attempt to integrate this compensation method into the operating conditions. These conditions included the newly installed local solenoid compensation for the CLEO detector magnetic field [11] and the use of eight trains of three bunches, as had been in operation for the preceding three months. At this time, the peak luminosity for eight trains of three bunches was $6.4 \times 10^{31} \mathrm{~cm}^{-2} \mathrm{~s}^{-1}$ and the peak total current in the two beams was $120 \mathrm{~mA}$. For the machine studies, it was decided to undertake a study for eight trains of four bunches starting with the same conditions, since this current distribution would reduce the (dominant) interaction point beam-beam interaction by $25 \%$ for the same total current. The studies began by characterizing the existing conditions for eight trains of four bunches per beam. A total two-beam current limit of $120 \mathrm{~mA}$ was reached when filling electrons against the stored positrons. This current limit during electron injection was the routine limitation observed during operations at this time and was due to the increased losses in the stored electron beam when the current increased and the pulsed injection orbit bumps were firing. The operating fractional horizontal and vertical tunes were 0.515 and 0.590 . The proximity of the horizontal tune to the half-integer resonance made the available operating horizontal tune range very narrow and required an adjustment of the horizontal tune systematically in one direction as the electron current increased and current limit was approached. This adjustment of the tune was needed to keep the zero-mode horizontal coherent beam-beam tune constant during filling.

After following the procedure for converting normal operating conditions into conditions having the partial compensation of the beam-beam interaction (see Sec. VI), the currents in the positron and electron beams were alternately raised to determine the new beam-beamcurrent limit. With the dynamic compensation of the beambeam interaction in operation, the first effect that was noted was that the horizontal tune adjustment, which was very critical during filling, was not needed, since this tune remained unchanged as the currents per bunch increased. This was the expected effect after compensating the local phase advance changes due to the beam-beam interaction. In practice, it was observed that the positron and electron injection conditions and colliding conditions required relatively little additional adjustment after starting the dynamic compensation. After about one hour of cycling through the injection and colliding conditions during the machine studies period, we were able to fill to a total current of $130 \mathrm{~mA}$ with reasonable lifetimes in collisions. Electron injection continued to be the limitation. At this point, the conditions were employed for the next seven hours during 
the initial return to luminosity-production operation. During this time, additional tuning permitted the total current to be raised to $144 \mathrm{~mA}$ with an increase in the peak luminosity to over $6.7 \times 10^{31} \mathrm{~cm}^{-2} \mathrm{~s}^{-1}$. Although these conditions looked promising in view of the brief time allotted to tuning, the filling of electrons continued to be slower than the normal operating conditions, owing in part to the fact that the injector for CESR-c had been tuned for three bunches per train and the filling rate for the fourth bunch was poor. Since the longer injection times would have reduced integrated luminosity, operating conditions were returned to eight trains of three bunches.

\section{CONCLUSION AND FUTURE DIRECTION}

As is true for other colliding-beam accelerators, the beam-beam interaction is one of the major performancelimiting effects for CESR-c. During multibunch operations, the maximum current per bunch falls far short of that observed for single-bunch operation. Modeling has shown the beam-beam interaction from the interaction point and parasitic crossings to contribute to the distortion of the optical functions with the greater effect in the horizontal plane owing to the working point proximity to the half-integer resonance. The reliability of the beambeam interaction modeling has been established by comparing calculated and measured orbit distortions at various values of bunch current. Additional confidence in the modeling has been obtained from the observation that the current limits during operations are correlated to a particular level of beta-function distortion in a variety of accelerator optics designs and that the calculated dynamic aperture is constricted to a particular limiting value as well. We draw the conclusion that the beam-beam interaction limits the operating currents when particles in the tails reach a loss rate sufficient to reduce either the beam lifetime or the injection rate.

Armed with the modeling of the beam-beam interaction, we have attempted to use the flexibility of the CESR-c optics to design a compensation algorithm to improve the loss rate for particles at large-amplitude oscillations. Employing quadrupole magnets in the neighborhood of each cluster of parasitic crossings, this compensation scheme reduces the effect of the beam-beam interaction from the parasitic crossings on the core of the counterrotating beam and partially compensates the beam-beam interaction effect from the main interaction point on the particles in the tails of the beam's distribution. The compensation of the parasitic crossings has been calculated using the model, while the compensation for the interaction point was determined empirically using single-bunch collisions. Machine studies have demonstrated an improvement in the total current in both beams of about $20 \%$ and an associated increase in the peak luminosity. Some aspects of this method are likely to be of interest at other colliders.
Future work will focus on two aspects of the beam-beam interaction current limit. The first is the evaluation of all new optics designs using the beam-beam interaction modeling methods developed for the design, assessment, and implementation of the partial compensation method. The second is to establish conditions that can be used for routine operations. The intention is to start with the accelerator conditions after they have been tuned extensively, yielding reproducible injection and luminosity performance. To these we will add the beam-beam interaction compensation, allowing an increase in the operating currents and luminosity. The difficulties observed with the transition to luminosity-production operations in the studies reported above were associated with an insufficient transition period to allow for the adjustments necessary to operations at higher current. A more deliberate plan for the next implementation will be required.

\section{ACKNOWLEDGMENTS}

The calculations and measurements described in this paper would not have been possible without the competent support of our colleagues in the CESR-c operations group. Jerry Codner, Mark Palmer, and John Sikora contributed to the execution of the machine studies and John Hylas provided essential operational expertise. Substantial assistance in the modeling came from David Rubin and David Sagan. David Rubin and Mike Forster incorporated beambeam interaction effects into the CESR-c lattice design procedure. We also wish to acknowledge the dedicated efforts of the CESR-c technical staff.

This work was supported by NSF Grant No. PHY0202078.

[1] J.M. Jowett, Handbook of Accelerator Physics and Engineering (World Scientific, Singapore, 1998), p. 144.

[2] P. Chen, Handbook of Accelerator Physics and Engineering (Ref. [1]), p. 140.

[3] N. Delerue, T. Tauchi, and K. Yokoya, Phys. Rev. ST Accel. Beams 8, 112801 (2005).

[4] D. Rice et al., in Proceedings of PAC'01 (2001), pp. 374376.

[5] J.A. Crittenden, M.G. Billing, and D.L. Rubin, in Proceedings of PAC'05 (2005), pp. 1275-1277.

[6] M. G. Billing, J.A. Crittenden, and M.A. Palmer, in Proceedings of PAC'05 (Ref. [5]), TPPP011.

[7] M. G. Billing and J. A. Crittenden, "Studies of the BeamBeam Interaction at CESR-c," in Proceedings of EPAC'06 (2006).

[8] D. Sagan, The BMAD Reference Manual, http:// www.lepp.cornell.edu/ dcs/bmad.

[9] M. Bassetti and G. A. Erskine, cern-isr-th/80-06, 1980.

[10] R. Talman, Physics of Particle Accelerators, AIP Conf. Proc. No. 153 (American Institute of Physics, New York, 1987), pp. 789-834.

[11] J. A. Crittenden, in Proceedings of EPAC'06 (Ref. [7]). 